\title{
Graphs Whose Certain Polynomials Have Few Distinct Roots
}

\author{
Saeid Alikhani \\ Department of Mathematics, Yazd University, P.O. Box 89195-741, Yazd, Iran \\ Correspondence should be addressed to Saeid Alikhani; alikhani206@gmail.com
}

Received 16 June 2013; Accepted 7 August 2013

Academic Editors: M. Chlebík and X. Yong

Copyright (C) 2013 Saeid Alikhani. This is an open access article distributed under the Creative Commons Attribution License, which permits unrestricted use, distribution, and reproduction in any medium, provided the original work is properly cited.

Let $G=(V, E)$ be a simple graph. Graph polynomials are a well-developed area useful for analyzing properties of graphs. We consider domination polynomial, matching polynomial, and edge cover polynomial of $G$. Graphs which their polynomials have few roots can sometimes give surprising information about the structure of the graph. This paper is primarily a survey of graphs whose domination polynomial, matching polynomial, and edge cover polynomial have few distinct roots. In addition, some new unpublished results and questions are concluded.

\section{Introduction}

Let $G=(V, E)$ be a simple graph. Graph polynomials are a well-developed area useful for analyzing properties of graphs.

We consider the domination polynomial, the matching polynomial (and the independence polynomial), and the edge cover polynomial of graph $G$. For convenience, the definition of these polynomials will be given in the following sections.

The corona of two graphs $G_{1}$ and $G_{2}$, as defined by Frucht and Harary in [1], is the graph $G=G_{1} \circ G_{2}$ formed from one copy of $G_{1}$ and $\left|V\left(G_{1}\right)\right|$ copies of $G_{2}$, where the $i$ th vertex of $G_{1}$ is adjacent to every vertex in the $i$ th copy of $G_{2}$. The corona $G \circ K_{1}$, in particular, is the graph constructed from a copy of $G$, where for each vertex $v \in V(G)$, a new vertex $v^{\prime}$ and a pendant edge $v v^{\prime}$ are added. The join of two graphs $G_{1}$ and $G_{2}$, denoted by $G_{1} \vee G_{2}$ is a graph with vertex set $V\left(G_{1}\right) \cup V\left(G_{2}\right)$ and edge set $E\left(G_{1}\right) \cup E\left(G_{2}\right) \cup\left\{u v \mid u \in V\left(G_{1}\right)\right.$ and $\left.v \in V\left(G_{2}\right)\right\}$.

The decycling number (or the feedback vertex number) of a graph $G$ is the minimum number of vertices that need to be removed in order to eliminate all its cycles.

The study of graphs whose polynomials have few roots can sometimes give surprising information about the structure of the graph. If $A$ is the adjacency matrix of $G$, then the eigenvalues of $A, \lambda_{1} \geq \lambda_{2} \geq \cdots \geq \lambda_{n}$ are said to be the eigenvalues of the graph $G$. These are the roots of the characteristic polynomial $\phi(G, \lambda)=\prod_{i=1}^{n}\left(\lambda-\lambda_{i}\right)$. For more details on the characteristic polynomials, see [2]. The characterization of graphs with few distinct roots of characteristic polynomials (i.e., graphs with few distinct eigenvalues) have been the subject of many researches. Graphs with three adjacency eigenvalues have been studied by Bridges and Mena [3] and Muzychuk and Klin [4]. Also van Dam studied graphs with three and four distinct eigenvalues [5-9]. Graphs with three distinct eigenvalues and index less than 8 were studied by Chuang and Omidi in [10].

This paper is primarily a survey of graphs whose polynomial domination, matching polynomial, and edge cover polynomial have few distinct roots. In addition, some new unpublished results and questions are concluded. In Section 2, we investigate graphs with few domination roots. In Section 3, we study graphs whose matching polynomials have few roots. Using these results we characterize line graphs whose independence polynomials have few roots. In Section 4, we characterize graphs with few edge cover roots. Finally in Section 5 , we state conjectures and open problems.

As usual, we use $\lfloor x\rfloor,\lceil x\rceil$ for the largest integer less than or equal to $x$, and for the smallest integer greater than or equal to $x$, respectively.

\section{Graphs with Few Domination Roots}

Let $G=(V, E)$ be a graph of order $|V|=n$. For any vertex $v \in V$, the open neighborhood of $v$ is the set $N(v)=\{u \in V \mid u v \in E\}$, and the closed neighborhood of $v$ is the set $N[v]=N(v) \cup\{v\}$. For a set $S \subseteq V$, the open 
neighborhood of $S$ is $N(S)=\bigcup_{v \in S} N(v)$, and the closed neighborhood of $S$ is $N[S]=N(S) \cup S$. A set $S \subseteq V$ is a dominating set if $N[S]=V$, or equivalently, every vertex in $V \backslash S$ is adjacent to at least one vertex in $S$. The domination number $\gamma(G)$ is the minimum cardinality of a dominating set in $G$. For a detailed treatment of this parameter, the reader is referred to [11]. Let $\mathscr{D}(G, i)$ be the family of dominating sets of a graph $G$ with cardinality $i$, and let $d(G, i)=|\mathscr{D}(G, i)|$. The domination polynomial $D(G, x)$ of $G$ is defined as $D(G, x)=$ $\sum_{i=0}^{|V(G)|} d(G, i) x^{i}[12-14]$. The path $P_{4}$ on 4 vertices, for example, has one dominating set of cardinality 4 , four dominating sets of cardinality 3 , and four dominating sets of cardinality 2 ; its domination polynomial is $D\left(P_{4}, x\right)=x^{4}+4 x^{3}+4 x^{2}$.

A root of $D(G, x)$ is called a domination root of $G$. In this section, the set of distinct roots of $D(G, x)$ is denoted by $Z(D(G, x))$.

A set $S \subseteq V$ is called an independent set if no pair of vertices of $S$ is adjacent. The independence number of graph $G$ is the size of a maximum independent set of $G$ and is denoted by $\alpha(G)$.

The following theorem prove that we have real domination roots of arbitrarily large modulus.

Theorem 1 (see [15]). The domination polynomial of the star graph, $D\left(K_{1, n}, x\right)$, where $n \in \mathbb{N}$, has a real root in the interval $(-2 n,-\ln (n))$, for $n$ sufficiently large.

The domination roots of $K_{1, n}$ for $1 \leq n \leq 60$ was shown in Figure 1.

In $[12,16]$ we have characterized graphs with one, two and three distinct domination roots. Since 0 is a root of any domination polynomial of graph $G$, we have the following theorem.

Theorem 2 (see [17]). A graph $G$ has one domination root if and only if $G$ is a union of isolated vertices.

It is interesting that all characterized graphs with two, three, and four distinct domination roots have special structures. As we will see, the structures of these graphs are the form of corona of two graphs. The following theorem characterizes graphs with two distinct domination roots.

Theorem 3 (see [12]). Let $G$ be a connected graph with exactly two distinct domination roots. Then there exists natural number $n$ such that $D(G, x)=x^{n}(x+2)^{n}$. Indeed $G=H \circ K_{1}$ for some graph $H$ of order $n$. Moreover, for every graph $H$ of order $n, D\left(H \circ K_{1}, x\right)=x^{n}(x+2)^{n}$.

Theorem 4 (see [12]). Let $G$ be a connected graph of order $n$. Then, $Z(D(G, x))=\{0,(-3 \pm \sqrt{5}) / 2\}$, if and only if $G=H \circ \bar{K}_{2}$, for some graph $H$. Indeed $D(G, x)=x^{n / 3}\left(x^{2}+3 x+1\right)^{n / 3}$.

Theorem 4 characterizes graphs with $Z(D(G, x))=$ $\{0,(-3 \pm \sqrt{5}) / 2\}$. These graphs are of the form $H \circ \bar{K}_{2}$, for some graph $H$ (see Figure 2).

For classifying all graphs with three distinct domination roots, we need to know that the value of the domination polynomial of every graph at -1 is nonzero (see [18] and Theorem 5).

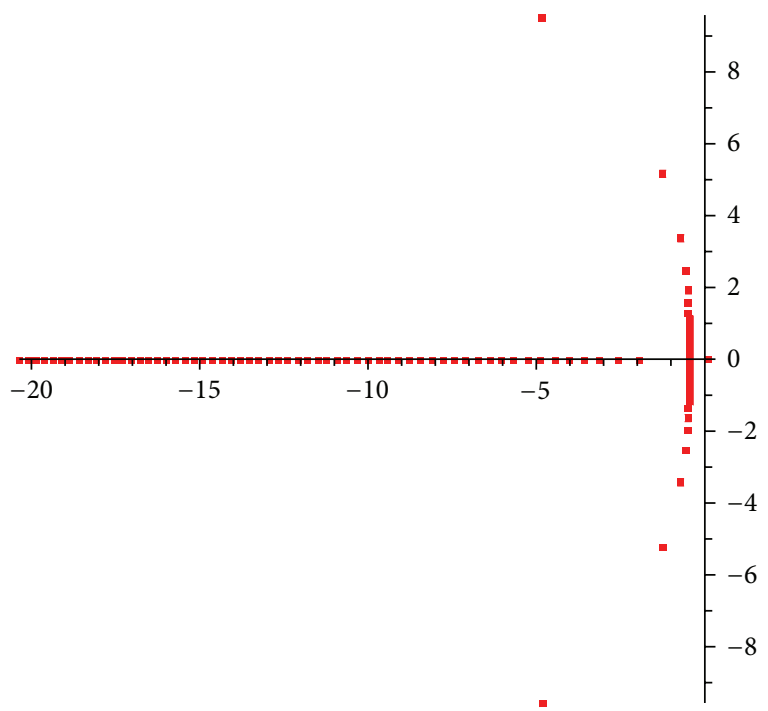

FIGURE 1: Domination roots of $K_{1, n}$ for $1 \leq n \leq 60$.

Theorem 5 (see [19]). Let $G$ be a graph. Then $D(G, r)$ is odd for every odd integer $r$. In particular, $D(G,-1)$ is odd.

The following corollary is an immediate consequence of Theorem 5.

Corollary 6 (see [19]). Every integer domination root of a graph $G$ is even.

The following theorem shows that roots of graphs with exactly three distinct domination roots cannot be any number.

Theorem 7 (see [12]). For every graph $G$ with exactly three distinct domination roots

$$
Z(D(G, x)) \subseteq\left\{0, \frac{-3 \pm \sqrt{5}}{2},-2 \pm \sqrt{2} i, \frac{-3 \pm \sqrt{3} i}{2}\right\} .
$$

Remark 8. Since $Z\left(D\left(K_{3}, x\right)\right)=\{0,(-3 \pm \sqrt{3} i) / 2\}$, $Z\left(D\left(P_{3}, x\right)\right)=\{0,(-3 \pm \sqrt{5}) / 2\}$, and $Z\left(D\left(C_{4}, x\right)\right)=\{0,-2 \pm$ $\sqrt{2} i\}$, for every number of set $\{0,(-3 \pm \sqrt{5}) / 2,-2 \pm \sqrt{2} i,(-3 \pm$ $\sqrt{3} i) / 2\}$, there exist a graph whose domination polynomials have exactly three distinct roots. Since cycles are determined by their domination polynomials [20], so graphs with exactly three domination roots from $\{0,(-3 \pm \sqrt{5}) / 2,-2 \pm \sqrt{2} i\}$ are $\mathrm{C}_{4}$ and are graphs of the form $\mathrm{H} \circ \bar{K}_{2}$, for graph $H$.

By Theorems 2, 3 and, 7, we have the following corollary.

Corollary 9 (see [16]). For every graph $G$ with at most three distinct domination roots

$$
Z(D(G, x)) \subseteq\left\{0,-2, \frac{-3 \pm \sqrt{5}}{2},-2 \pm \sqrt{2} i, \frac{-3 \pm \sqrt{3} i}{2}\right\} .
$$

Now we will study graphs with exactly four distinct domination roots. 


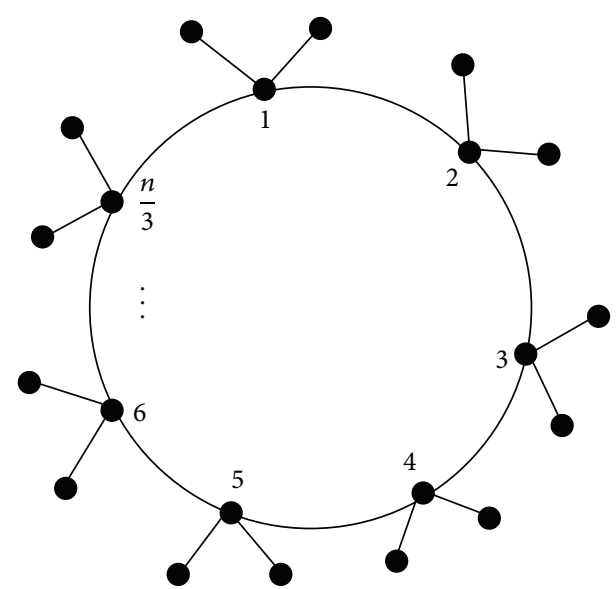

FIGURE 2: Graph with $D(G, x)=x^{[n / 3\rceil}\left(x^{2}+3 x+1\right)^{[n / 3]}$.

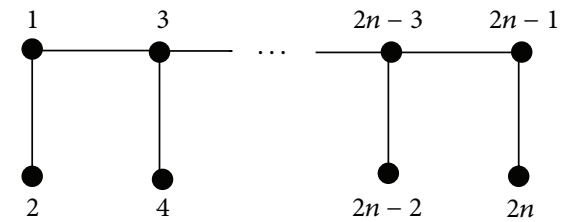

Figure 3: Labeled centipede, $P_{n}^{*}$.

Let $G_{n}$ be an arbitrary graph of order $n$. Let it denote the graph $G_{n} \circ K_{1}$ simply by $G_{n}^{*}$. Here we consider the labeled $G_{n}^{*}$ as shown in Figure 3 (the graph in this figure is $P_{n}^{*}$ which is called centipede). We denote the graph obtained from $G_{n}^{*}$ by deleting the vertex labeled $2 n$ as $G_{n}^{*}-\{2 n\}$.

The following theorem states a recursive formula for the domination polynomial of $G_{n}^{*}-\{2 n\}$.

Theorem 10 (see [21]). For every $n \geq 5$,

$$
\begin{aligned}
D\left(G_{n}^{*}-\{2 n\}, x\right)= & x\left[D\left(G_{n-1}^{*}, x\right)+D\left(G_{n-2}^{*}, x\right)\right] \\
& +x^{2} D\left(G_{n-2}^{*}, x\right) .
\end{aligned}
$$
$\{2 n\}, x)$.

The following theorem gives the formula for $D\left(G_{n}^{*}-\right.$

Theorem 11 (see [21]). For every $n \geq 2, D\left(G_{n}^{*}-\{2 n\}, x\right)=$ $\left(x^{2}+3 x+1\right) x^{n-1}(x+2)^{n-2}$.

The following theorem characterizes graphs with four domination roots $-2,0,(-3 \pm \sqrt{5}) / 2$.

Theorem 12 (see [21]). Let $G$ be a connected graph of order $n$. Then, $Z(D(G, x))=\{-2,0,(-3 \pm \sqrt{5}) / 2\}$, if and only if $G=$ $G_{n / 2}^{*}-\{n\}$, for some graph $G_{n / 2}$ of order $n / 2$. Indeed

$$
D(G, x)=\left(x^{2}+3 x+1\right) x^{(n / 2)-1}(x+2)^{(n / 2)-2} .
$$

Since domination polynomial is monic polynomial with integer coefficients, its zeros are algebraic integers. This naturally raises the question: which algebraic integers can occur as zeros of domination polynomials? (see [22]). Using tables of domination polynomials (see [23]), we think that the number of algebraic integers which can be roots of graphs with exactly four distinct domination roots are about nine numbers, but we are not able to prove it. So complete characterization of graphs with exactly four distinct domination roots remains as an open problem. In other words, we have the following question.

Question 1. Which algebraic integers are domination roots of graphs with exactly four distinct domination roots?

All real roots of the domination polynomial are necessarily negative, but it is not surprising that for some graphs there exists imaginary roots with positive real parts [24]. Surprisingly, in [15] it has shown that the domination roots are in fact dense in $\mathbb{C}$.

Theorem 13 (see [15]). The closure of the domination roots is the whole complex plane.

\section{Graphs with Few Matching Roots}

In this section, we study graphs whose matching polynomials have few roots. First, we state the definition of matching polynomial. Let $G=(V, E)$ be a graph of order $n$ and size $m$. An $r$-matching of $G$ is a set of $r$ edges of $G$ where no two of them have common vertex. The maximum number of edges in the matching of a graph $G$ is called the matching number of $G$ and is denoted by $\alpha^{\prime}(G)$. The matching polynomial is defined by

$$
\mu(G, x)=\sum_{k=0}^{\lfloor n / 2\rfloor}(-1)^{k} m(G, k) x^{n-2 k}
$$

where $m(G, k)$ is the number of $k$-matching of $G$, and $m(G, 0)=1$. The roots of $\mu(G, x)$ are called the matching roots of $G$. As an example, the matching polynomial of path $P_{5}$ is $\mu\left(P_{5}, x\right)=(x-1) x(x+1)\left(x^{2}-3\right)$. For more details of this polynomial refer to [25-27].

Two following theorems are the first results on matching roots.

Theorem 14 (see [28]). The roots of matching polynomial of any graph are all real numbers.

Theorem 15 (see [29]). If $G$ has a Hamiltonian path, then all roots of its matching polynomial are simple (have multiplicity 1).

We need the following definition to study graphs with few matching roots.

Add a single vertex $u$ to the graph $r K_{1, k} \cup t K_{1}$ and join $u$ to the other vertices by $p+q$ edges so that the resulting graph is connected and $u$ is adjacent with $q$ centers of the stars (for $K_{1,1}$ either of the vertices may be considered as a center). We denote the resulting graph by $\mathcal{S}(r, k, t ; p, q)$ (see Figure 4$)$. Clearly $r+t \leq p+q \leq r(k+1)+t$ and $0 \leq q \leq r$ (see [30]). 


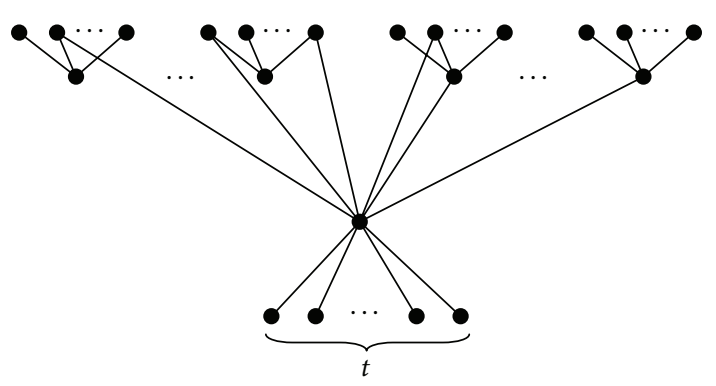

Figure 4: A typical graph in the family $\delta(r, k, t ; p, q)$ : the central vertex has degree $p$ and it is joined to $q$ centers of the stars $K_{1, k}$.

For any $G \in \mathcal{S}(r, 3, t ; p, q)$, we add $s$ copies of $K_{3}$ to $G$ and join them by $l$ edges to the vertex $u$ of $G$. Clearly $s \leq l \leq 3 s$. We denote the set of these graphs by $\mathscr{H}(r, s, t ; p, q, l)$.

The following theorem gives the matching polynomial of graph $G$ in the family $\mathcal{S}(r, k, t ; p, q)$.

Theorem 16 (see [30]). For every $G \in \mathcal{S}(r, k, t ; p, q)$,

$$
\begin{aligned}
\mu(G, x)= & x^{r(k-1)+t-1}\left(x^{2}-k\right)^{r-1} \\
& \times\left(x^{4}-(p+k) x^{2}+(p-q)(k-1)+t\right) .
\end{aligned}
$$

Theorem 17 (see [30]). For every $G \in \mathscr{H}(r, s, t ; p, q, l)$,

$$
\begin{aligned}
\mu(G, x)= & x^{2 r+s+t-1}\left(x^{2}-3\right)^{r+s-1} \\
& \times\left(x^{4}-(p+l+3) x^{2}+3 t+2(p-t-q)+l\right) .
\end{aligned}
$$

Similar to [30], we distinguish some special graphs in the families $S$ and $H$ which are important for our study.

We denote the family $\mathcal{S}(r, 1,0 ; s, q)$ which consists of a single graph by $S(r, s)$. Note that in this case $q$ is determined by $r$ and $s$; namely, $q=s-r$. Its matching polynomial is

$$
\mu(S(r, s), x)=x\left(x^{2}-s-1\right)\left(x^{2}-1\right)^{r-1} .
$$

The family $\mathcal{S}(r, k, 0 ; r, r)$ consists of a single graph which is denoted by $T(r, k)$. Its matching polynomial is

$$
\mu(T(r, k), x)=x^{r(k-1)+1}\left(x^{2}-r-k\right)\left(x^{2}-k\right)^{r-1} .
$$

We also denote the unique graphs in $\mathcal{S}(1, k, t ; l+t, 0)$ and $\mathcal{S}(1, k, t ; l+t+1,1)$ by $K(k, t ; l)$ and $K^{\prime}(k, t ; l)$, respectively. Their matching polynomials are

$$
\begin{aligned}
& \mu(K(k, t ; l), x) \\
& \quad=x^{k+t-2}\left(x^{4}-(k+t+l) x^{2}+(l+t)(k-1)+t\right), \\
& \mu\left(K^{\prime}(k, t ; l), x\right) \\
& \quad=x^{k+t-2}\left(x^{4}-(k+t+l+1) x^{2}+(l+t)(k-1)+t\right) .
\end{aligned}
$$

Moreover, we denote the unique graph in $\mathscr{H}(0,1, t ; t, 0, l)$ by $L(t, l)$ for $l=1,2,3$. We have $\mu(L(t, l), x)=x^{t}\left(x^{4}-(t+l+\right.$ 3) $\left.x^{2}+3 t+l\right)$. Typical graphs from the above families are shown in Figure 5.

Theorem 18 (see [30]). Let $G$ be a connected graph, and let $z(G)$ be the number of its distinct matching roots.

(i) If $z(G)=2$, then $G \simeq K_{2}$.

(ii) If $z(G)=3$, then $G$ is either a star or $K_{3}$.

(iii) If $z(G)=4$, then $G$ is a nonstar graph with 4 vertices.

(iv) If $z(G)=5$, then $G$ is one of the graphs $K(k, t ; l)$, $K^{\prime}(k, t ; l), L(t ; l), T(r, k)$, and $S(r, s)$, for some integers $k, r, s, t$, and $l$ or a connected nonstar graph with 5 vertices.

Using the previous theorem we would like to study graphs with few independence roots. First, we recall the definition of independence polynomial.

An independent set of a graph $G$ is a set of vertices where no two vertices are adjacent. The independence number is the size of a maximum independent set in the graph and is denoted by $\alpha(G)$. For a graph $G$, let $i_{k}$ denote the number of independent sets of cardinality $k$ in $G(k=0,1, \ldots, \alpha)$. The independence polynomial of $G$,

$$
I(G, x)=\sum_{k=0}^{\alpha} i_{k} x^{k}
$$

is the generating polynomial for the independent sequence $\left(i_{0}, i_{1}, i_{2}, \ldots, i_{\alpha}\right)$. For further studies on independence polynomial and independence root refer to [31-33].

The path $P_{4}$ on 4 vertices, for example, has one independent set of cardinality 0 (the empty set), four independent sets of cardinality 1 , and three independent sets of cardinality 2 ; its independence polynomial is then $I\left(P_{4}, x\right)=1+4 x+3 x^{2}$.

Here we recall the definition of line graph. Given a graph $H=(V, E)$, the line graph of $H$, denoted by $L(H)$, is a graph with vertex set $E$; two vertices of $L(H)$ are adjacent if and only if the corresponding edges in $H$ share at least one endpoint. We say that $G$ is a line graph if there is a graph $H$ for which $G=L(H)$.

Theorem 19 (see [34]). For every graph $G, \mu(G, x)=$ $x^{n} I\left(L(G),-1 / x^{2}\right)$.

The following corollary is an immediate consequence of Theorem 19.

Corollary 20. If $\alpha \neq 0$ is a matching root of $G$, then $-1 / \alpha^{2}$ is an independence root of $L(G)$.

Now we are ready to state a theorem for graphs whose independence polynomials have few roots. The following theorem follows from Theorem 19 and Corollary 20. 

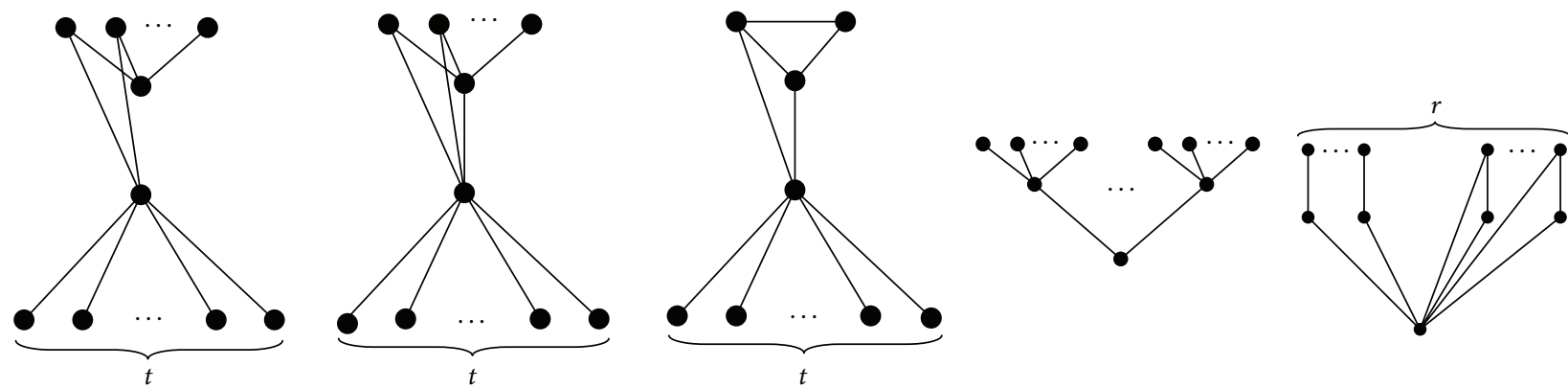

Figure 5: The graphs $K(k, t ; l), K^{\prime}(k, t ; l), L(t, l), T(r, k)$, and $S(r, s)$, respectively.

Theorem 21. Let $G$ be a connected graph and $z(L(G))$ be the number of distinct independence nonzero roots of $L(G)$.

(i) If $z(L(G))=2$, then $L(G) \simeq K_{2}$.

(ii) If $z(L(G))=3$, then $L(G)$ is either a star or $K_{3}$.

(iii) If $z(L(G))=4$, then $L(G)$ is a nonstar graph with 4 vertices.

(iv) If $z(L(G))=5$, then $L(G)$ is one of the graphs and $G$ is one of the graphs $K(k, t ; l), K^{\prime}(k, t ; l), L(t ; l), T(r, k)$, and $S(r, s)$, for some integers $k, r, s, t$, and $l$ or a connected nonstar graph with 5 vertices.

Here we bring the independence roots of families of paths and cycles. In the next section, we compare these roots with roots of edge cover polynomials of paths and cycles.

Theorem 22 (see [31]). (i) For any integer $n, I\left(P_{n}, x\right)$ has the following zeros,

$$
p_{s}^{(n)}=-\frac{1}{2(1+\cos (2 s \pi /(n+2)))}, \quad s=1,2, \ldots,\left\lfloor\frac{n+1}{2}\right\rfloor .
$$

(ii) For any integer $n \geq 3, I\left(C_{n}, x\right)$ has the following zeros,

$$
c_{s}^{(n)}=-\frac{1}{2(1+\cos (((2 s-1) \pi) / n))}, \quad s=1,2, \ldots,\left\lfloor\frac{n}{2}\right\rfloor .
$$

Corollary 23 (see [31]). The independence roots of the family $\left\{P_{n}\right\}$ and $\left\{C_{n}\right\}$ are real and dense in $(-\infty,-1 / 4]$.

In [35] the authors studied graphs whose independence roots are rational.

Theorem 24 (see [35]). Let $G$ be a graph with rational polynomial $I(G, x)$. If $i(G, r-1)>i(G, r)$, for some $r, 1 \leq$ $r \leq \alpha(G)$, then $G$ has an independence root in the interval $(-1,-1 / \alpha(G)]$.

The following theorem characterizes graphs with exactly one independence root.

Theorem 25 (see [35]). Let $G$ be a graph of order $n$. Then $I(G, x)$ has exactly one root if and only if $G=r K_{s}$, where $n=r s$ for some natural $r, s$.
Theorem 26 (see [36]). For any graph $G,|I(G,-1)| \leq 2^{\nu(G)}$, where $\nu(G)$ is the decycling number of $G$.

\section{Graphs with Few Edge-Cover Roots}

In this section, we characterize graphs whose edge cover polynomials have one and two distinct roots. First, we state the definition of edge-cover polynomial of a graph.

For every graph $G$ with no isolated vertex, an edge covering of $G$ is a set of edges of $G$ such that every vertex is incident to at least one edge of the set. A minimum edge covering is an edge cover of the smallest possible size. The edge covering number of $G$ is the size of a minimum edge cover of $G$ and is denoted by $\rho(G)$. The edge cover polynomial of $G$ is the polynomial $E(G, x)=\sum_{i=1}^{m} e(G, i) x^{i}$, where $e(G, i)$ is the number of edge covering sets of $G$ of size $i$. Note that if graph $G$ has isolated vertex then we put $E(G, x)=0$ and if $V(G)=E(G)=\emptyset$, then $E(G, x)=1$. For more detail on this polynomial refer to $[37,38]$.

As an example, the edge cover polynomial of path $P_{5}$ is $E\left(P_{5}, x\right)=2 x^{3}+x^{4}$. Also $E\left(K_{1, n}, x\right)=x^{n}$. As usual we use $\delta(G)$ to denote the minimum degree of graph $G$.

The following theorem is a recursive formula for edge cover polynomial of a graph.

Theorem 27 (see [37]). Let $G$ be a graph, $u, v \in V(G)$ and $u v$ be an edge of $G$. Then

$$
\begin{aligned}
E(G, x)= & (x+1) E(G \backslash u v, x) \\
& +x(E(G \backslash u, x)+E(G \backslash v, x)+E(G \backslash\{u, v\})) .
\end{aligned}
$$

The following results are about edge cover polynomial of a graph.

Lemma 28 (see [37]). Let $G$ be a graph of order $n$ and size $m$ with no isolated vertex. If the edge cover polynomial of $G$ is $E(G, x)=\sum_{i=\rho(G)}^{m} e(G, i) x^{i}$, then the following hold:

(i) $n \leq 2 \rho(G)$.

(ii) If $i_{0}=\min \left\{i \mid e(G, i)=\left(\begin{array}{c}m \\ i\end{array}\right)\right\}$, then $\delta=m-i_{0}+1$.

(iii) If $G$ does not have connected component isomorphic to $K_{2}$, then $a_{\delta(G)}=\left(\begin{array}{c}m \\ m-\delta\end{array}\right)-e(G, m-\delta)$. 


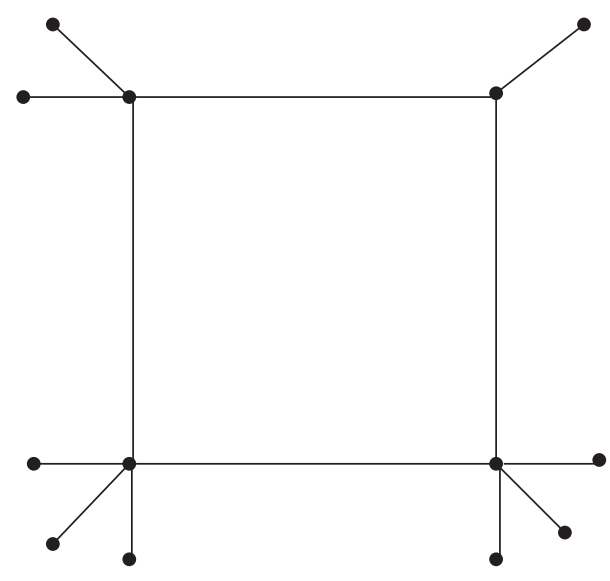

Figure 6: The graph $C_{4}(9)$.

Theorem 29 (see [38]). Let $G$ be a graph. Then $E(G, x)$ has at least $\delta(G)-2$ nonreal roots (not necessary distinct). In particular, when $\delta(G)=3, E(G, x)$ has at least two distinct nonreal roots.

Now, we will characterize graphs with few edge cover roots. Note that zero is one of the roots of $E(G, x)$ with multiplicity $\rho(G)$. The next theorem characterizes all graph $G$ whose edge cover polynomials have exactly one distinct root. Note that $E\left(K_{1, n}, x\right)=x^{n}$.

Theorem 30 (see [38]). Let $G$ be a graph. Then $E(G, x)$ has exactly one distinct root if and only if every connected component of $G$ is star.

We need the following definition to study graphs with two distinct edge cover roots.

Let $H$ be a graph of order $n$ and size $m$. Suppose $\left\{v_{1}, \ldots, v_{n}\right\}$ is the vertex set of $H$. By $H(r)$ we mean the graph obtained by joining $r_{i} \geq 1$ pendant vertices to vertex $v_{i}$, for $i=1, \ldots n$ such that $\sum r_{i}=r$. If $m$ is the size of $H$, then $H(r)$ is a graph of order $n+r$ and size $m+r$. The graph $C_{4}(9)$ shown in Figure 6.

Theorem 31 (see [38]). Let $G$ be a graph. Then $E(G, x)=$ $x^{r}(x+1)^{m}$, for some natural numbers $r$ and $m$, if and only if there exists a graph $H$ with size $m$ such that $G=H(r)$.

The next theorem characterizes all graphs $G$ for which $E(G, x)$ has exactly two distinct roots.

Theorem 32 (see [38]). Let $G$ be a connected graph whose edge cover polynomial has exactly two distinct roots. Then one of the following holds:

(i) $G=H(r)$, for some connected graph $H$ and natural number $r$.

(ii) $G=K_{3}$.

(iii) $\delta(G)=1, E(G, x)=x^{(m+s) / 2}(x+2)^{(m-s) / 2}$, where $s$ is the number of pendant vertices of $G$. (iv) $\delta(G)=2, E(G, x)=x^{m / 2}(x+2)^{m / 2}, a_{2}(G)=m / 2$, and $G$ has cycle of length 3 or 5 .

By Theorems 31 and 32 we have the following corollaries.

Corollary 33 (see [37]). Let $G$ be a connected graph. If $E(G, x)$ has exactly two distinct roots, then $Z(E(G, x))=\{-1,0\}$, $Z(E(G, x))=\{-2,0\}$, or $Z(E(G, x))=\{-3,0\}$. Also $\delta(G)=1$ or $\delta(G)=2$.

Corollary 34 (see [37]). Let $G$ be a connected graph. If $Z(E(G, x))=Z\left(E\left(K_{3}, x\right)\right)=\{-3,0\}$ if and only if $G=K_{3}$.

We have the following corollary.

Corollary 35. Let $\mathrm{G}$ be a graph with at most two distinct edge cover roots. Then $Z(E(G, x)) \subseteq\{-3,-2,-1,0\}$.

There are infinite graphs $G$ with $\delta(G)=1$ and $Z(E(G, x)) \subseteq\{-2,-1,0\}$. To see some algorithms for the constructing of these kind of graphs refer to [37]. For the case $\delta(G)=2$, we can see that if $G$ is a graph with $\delta(G)=2$ and $Z(E(G, x))=\{-2,0\}$, then $G$ has cycle with length 3 or 5 (see [37]). For this case there is the following conjecture.

Conjecture 1 (see [37]). There isn't any graph $G$ with $\delta(G)=2$ and $Z(E(G, x))=\{-2,0\}$.

It is proven that if $G$ is a graph without cycle of length 3 or 5 and $\delta(G)=2$, then $E(G, x)$ has at least three distinct roots (see [37]). Theorem 32 and previous conjecture implies that if $\delta(G)=2$ and $G \neq K_{3}$, then $E(G, x)$ has at least three distinct roots.

The following theorem gives the roots of edge cover polynomial of paths and cycles.

Theorem 36 (see [38]). (i) For any integer $n, E\left(P_{n}, x\right)$ has the following nonzero roots:

$$
-2\left(1+\cos \frac{2 s \pi}{n-1}\right), \quad s=1,2, \ldots,\left\lfloor\frac{n}{2}\right\rfloor-1 .
$$

(ii) For any integer $n \geq 3, E\left(C_{n}, x\right)$ has the following zeros:

$$
c_{s}^{(n)}=-2\left(1+\cos \frac{(2 s+1) \pi}{n}\right), \quad s=0,1, \ldots,\left\lfloor\frac{n}{2}\right\rfloor-1 .
$$

Also we have the following theorem for roots of $E\left(P_{n}, x\right)$ and $E\left(C_{n}, x\right)$.

Theorem 37 (see [38]). The roots of the family $\left\{E\left(P_{n}, x\right)\right\}$ and $\left\{E\left(C_{n}, x\right)\right\}$ are real and dense in $(-4,0]$.

Remark 38. It is interesting that the nonzeros roots of $I\left(C_{n}, x\right)$ are exactly the inverse of (nonzeros) roots of $E\left(C_{n}, x\right)$.

Note that in [38], it is proved that if every block of the graph $G$ is $K_{2}$ or a cycle, then all real roots of $E(G, x)$ are in the interval $(-4,0]$.

Since for every $n, \sqrt{n}$ is a root of the characteristic polynomial of $K_{1, n}$, we can conclude that there is no constant 
bound for the roots of the characteristic polynomials and matching polynomials. Sokal in [39], proved that for every graph $G$, the absolute value of any root of the chromatic polynomial of $G$ is at most $8 \Delta(G)$, where $\Delta(G)$ denotes the maximum degree of $G$. On the other hand, $\chi(G)-1$ is clearly a root of the chromatic polynomial of $G$, where $\chi(G)$ denotes the chromatic number of $G$. Therefore, there is no constant bound for the roots of chromatic polynomials. For more details on the chromatic polynomials, see [40]. Surprisingly, in the following theorem we observe that there is a constant bound for the roots of the edge cover polynomials.

Theorem 39 (see [38]). All roots of the edge cover polynomial lie in the ball

$$
\left\{z \in \mathbb{C}:|z|<\frac{(2+\sqrt{3})^{2}}{1+\sqrt{3}} \simeq 5.099\right\} .
$$

Here, we would like to study the edge cover polynomial of a graph $G$ at -1 . We need the following theorem.

Theorem 40 (see [38]). Let $G$ be a graph. Then $E(G, x)=$ $\sum_{S \subseteq V(G)}(-1)^{|S|}(x+1)^{|E(G \mid S)|}$.

Theorem 41. Let $G$ be a graph of order $n$. Then $E(G,-1)=$ $(-1)^{n} I(G,-1)$.

Proof. Clearly, $E(G \backslash S)=0$ implies that $V(G) \backslash S$ is an independent set of $G$ and vice versa. So by Theorem 40 , $E(G,-1)=(-1)^{n} I(G,-1)$.

By Theorems 26 and 41 we have the following result.

Theorem 42 (see [37]). For every graph $G,|E(G,-1)| \leq 2^{\nu(G)}$, where $v(G)$ is the decycling number of $G$.

\section{Open Problems and Conjectures}

In this section, we state and review some open problems and conjectures related to the subject of this paper.

We have characterized all graphs with exactly three distinct domination roots and proved that for any graph $G$ with exactly three distinct domination roots, we have

$$
Z(D(G, x)) \subseteq\left\{0, \frac{-3 \pm \sqrt{5}}{2},-2 \pm \sqrt{2} i, \frac{-3 \pm \sqrt{3} i}{2}\right\} .
$$

The following problem remains as an open problem.

Problem 1 (see [16]). Characterize all graphs with exactly three distinct domination roots $\{0,(-3 \pm \sqrt{3} i) / 2\}$.

We characterized graphs with four distinct roots $Z(D(G, x))=\{-2,0,(-3 \pm \sqrt{5}) / 2\}$. We think that the number of algebraic integers which can be roots of graphs with exactly four distinct domination roots are about nine numbers, but we couldn't prove it yet. So complete characterization of graphs with exactly four distinct domination roots remains as an open problem. In other words, we have two following questions regarding graphs with exactly four distinct domination roots.

Problem 2. Which algebraic integers are domination roots of graphs with exactly four distinct domination roots?

Problem 3 (see [21]). Characterize all graphs with exactly four distinct domination roots.

There are graphs with no nonzero real domination roots except zero. As examples for every even $n$, no nonzero real numbers are domination roots of $K_{n, n}$, and no nonzero real numbers are domination roots of Dutch Windmill graphs (see [24]). So the following question is an interesting problem.

Problem 4 (see [16]). Characterize all graphs with no real domination roots except zero.

Corollary 6 implies that every integer domination root of a graph is even. We recall the following conjecture.

Conjecture 2 (see [16]). If $r$ is an integer domination root of a graph, then $r=0$ or $r=-2$.

Conjecture 3. Real roots of the families $D\left(P_{n}, x\right)$ and $D\left(C_{n}, x\right)$ are dense in the interval $[-2,0]$, for $n \geq 4$.

Theorem 25 implies that for a graph of order $n, I(G, x)$ has exactly one root if and only if $G=r K_{s}$, where $n=r s$ for some natural $r, s$. Also Theorem 21 characterizes line graphs whose independence polynomials have at most five distinct roots. Therefore, complete characterization of graphs with few independence roots remains as an open problem.

Problem 5. Characterize all graphs with few independence roots.

The following conjectures are related to the roots of edge cover polynomial of a graph.

Conjecture 4 (see [37]). If $\delta(G)=2$ and $G \neq K_{3}$, then $E(G, x)$ has at least three distinct roots.

Conjecture 5 (see [37]). Let $G$ be a graph. Then $E(G, x)$ has at least $\delta(G)$ distinct roots.

Conjecture 6 (see [38]). Let $G$ be a graph with $\delta(G)=2$. If $E(G, x)$ has only real roots, then all connected components of $G$ are cycles.

\section{Acknowledgment}

The author would like to express his gratitude to the referees for their comments.

\section{References}

[1] R. Frucht and F. Harary, "On the corona of two graphs," Aequationes Mathematicae, vol. 4, pp. 322-325, 1970. 
[2] D. M. Cvetković, M. Doob, and H. Sachs, Spectra of Graphs: Theory and Applications, Johann Ambrosius Barth, Heidelberg, Germany, 3rd edition, 1995.

[3] W. G. Bridges and R. A. Mena, "Multiplicative cones-a family of three eigenvalue graphs," Aequationes Mathematicae, vol. 22, no. 2-3, pp. 208-214, 1981.

[4] M. Muzychuk and M. Klin, "On graphs with three eigenvalues," Discrete Mathematics, vol. 189, no. 1-3, pp. 191-207, 1998.

[5] E. R. van Dam, "Regular graphs with four eigenvalues," Linear Algebra and Its Applications, vol. 226-228, pp. 139-162, 1995.

[6] E. R. van Dam, Graphs with few eigenvalues-an interplay between combinatorics and algebra [Ph.D. thesis], Center Dissertation Series 20, Tilburg University, 1996.

[7] E. R. van Dam, "Nonregular graphs with three eigenvalues," Journal of Combinatorial Theory. Series B, vol. 73, no. 2, pp. 101118, 1998.

[8] E. R. van Dam and W. H. Haemers, "Which graphs are determined by their spectrum?" Linear Algebra and Its Applications, vol. 373, pp. 241-272, 2003.

[9] E. R. van Dam and E. Spence, "Small regular graphs with four eigenvalues," Discrete Mathematics, vol. 189, no. 1-3, pp. 233-257, 1998.

[10] H. Chuang and G. R. Omidi, "Graphs with three distinct eigenvalues and largest eigenvalues less than 8," Linear Algebra and Its Applications, vol. 430, no. 8-9, pp. 2053-2062, 2009.

[11] T. W. Haynes, S. T. Hedetniemi, and P. J. Slater, Fundamentals of Domination in Graphs, vol. 208 of Monographs and Textbooks in Pure and Applied Mathematics, Marcel Dekker, New York, NY, USA, 1998.

[12] S. Alikhani and Y. H. Peng, "Introduction to domination polynomial of a graph," Ars Combinatoria, http://arxiv.org/abs/0905 .2251.

[13] S. Alikhani and Y. Peng, "Dominating sets and domination polynomials of certain graphs. II," Opuscula Mathematica, vol. 30, no. 1, pp. 37-51, 2010.

[14] S. Alikhani and Y.-H. Peng, "Dominating sets and domination polynomials of paths," International Journal of Mathematics and Mathematical Sciences, vol. 2009, Article ID 542040, 10 pages, 2009.

[15] J. Brown and J. Tufts, "On the roots of domination polynomials," Graphs and Combinatorics, 2013.

[16] S. Akbari, S. Alikhani, M. R. Oboudi, and Y. H. Peng, "On the zeros of domination polynomial of a graph," in Combinatorics and Graphs, vol. 531, pp. 109-115, American Mathematical Society, Providence, RI, USA, 2010.

[17] S. Akbari, S. Alikhani, and Y.-h. Peng, "Characterization of graphs using domination polynomials," European Journal of Combinatorics, vol. 31, no. 7, pp. 1714-1724, 2010.

[18] A. E. Brouwer, "The number of dominating sets of a finite graph is odd," preprint.

[19] S. Alikhani, "The domination polynomial of a graph at-1," Graphs and Combinatorics, vol. 29, no. 5, pp. 1175-1181, 2013.

[20] S. Akbari and M. R. Oboudi, "Cycles aredetermined by their domination polynomials," Ars Combinatoria, http://arxiv.org/ abs/0908.3305.

[21] S. Alikhani, "On the graphs with four distinct domination roots," International Journal of Computer Mathematics, vol. 88, no. 13, pp. 2717-2720, 2011.

[22] S. Alikhani and R. Hasni, "Algebraic integers as chromatic and domination roots," International Journal of Combinatorics, vol. 2012, Article ID 780765, 8 pages, 2012.
[23] S. Alikhani, Dominating sets and domination polynomials of graphs [Ph.D. thesis], Universiti Putra Malaysia, 2009.

[24] S. Alikhani and E. Deutsch, "New results on domination polynomals and domination roots," submitted.

[25] E. J. Farrell, "On a general class of graph polynomials," Journal of Combinatorial Theory. Series B, vol. 26, no. 1, pp. 111-122, 1979.

[26] E. J. Farrell, "An introduction to matching polynomials," Journal of Combinatorial Theory. Series B, vol. 27, no. 1, pp. 75-86, 1979.

[27] C. D. Godsil, "Algebraic matching theory," Electronic Journal of Combinatorics, vol. 2, article R8, 1995.

[28] O. J. Heilmann and E. H. Lieb, "Monomers and dimers," Physical Review Letters, vol. 24, no. 25, pp. 1412-1414, 1970.

[29] C. D. Godsil, Algebraic Combinatorics, Chapman and Hall Mathematics Series, Chapman \& Hall, New York, NY, USA, 1993.

[30] E. Ghorbani, "Graphs with few matching roots," Graphs and Combinatorics, vol. 29, no. 5, pp. 1377-1389, 2013.

[31] S. Alikhani and Y. Peng, "Independence roots and independence fractals of certain graphs," Journal of Applied Mathematics and Computing, vol. 36, no. 1-2, pp. 89-100, 2011.

[32] J. I. Brown, C. A. Hickman, and R. J. Nowakowski, "On the location of roots of independence polynomials," Journal of Algebraic Combinatorics, vol. 19, no. 3, pp. 273-282, 2004.

[33] J. I. Brown, K. Dilcher, and R. J. Nowakowski, "Roots of independence polynomials of well covered graphs," Journal of Algebraic Combinatorics, vol. 11, no. 3, pp. 197-210, 2000.

[34] C. D. Godsil and I. Gutman, "On the theory of the matching polynomial," Journal of Graph Theory, vol. 5, no. 2, pp. 137-144, 1981.

[35] S. Akbari, M. R. Oboudi, and S. Qajar, "On the rational independence roots," Contemporary Mathematics, vol. 531, pp. 149-158, 2010.

[36] V. E. Levit and E. Mandrescu, “The independence polynomial of a graph at -1," http://arxiv.org/abs/0904.4819v1.

[37] S. Akbari and M. R. Oboudi, "On the edge cover polynomial of a graph," European Journal of Combinatorics, vol. 34, no. 2, pp. 297-321, 2013.

[38] P. Csikvári and M. R. Oboudi, "On the roots of edge cover polynomials of graphs," European Journal of Combinatorics, vol. 32, no. 8, pp. 1407-1416, 2011.

[39] A. D. Sokal, "Bounds on the complex zeros of (di)chromatic polynomials and Potts-model partition functions," Combinatorics, Probability and Computing, vol. 10, no. 1, pp. 41-77, 2001.

[40] F. M. Dong, K. M. Koh, and K. L. Teo, Chromatic Polynomials and Chromaticity of Graphs, World Scientific Publishing, Hackensack, NJ, USA, 2005. 


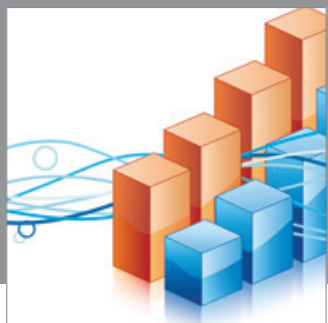

Advances in

Operations Research

mansans

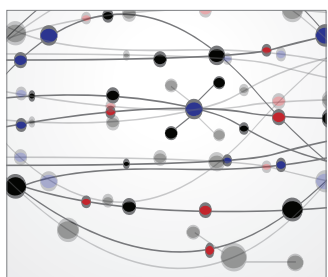

The Scientific World Journal
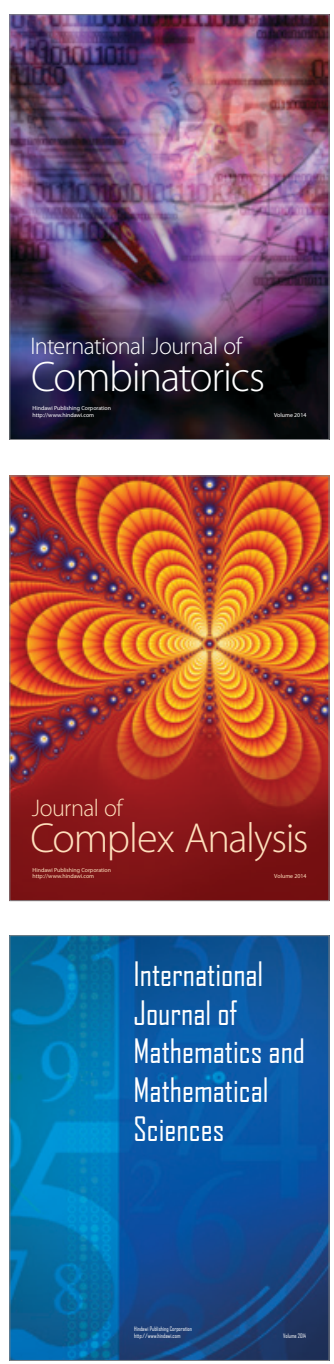
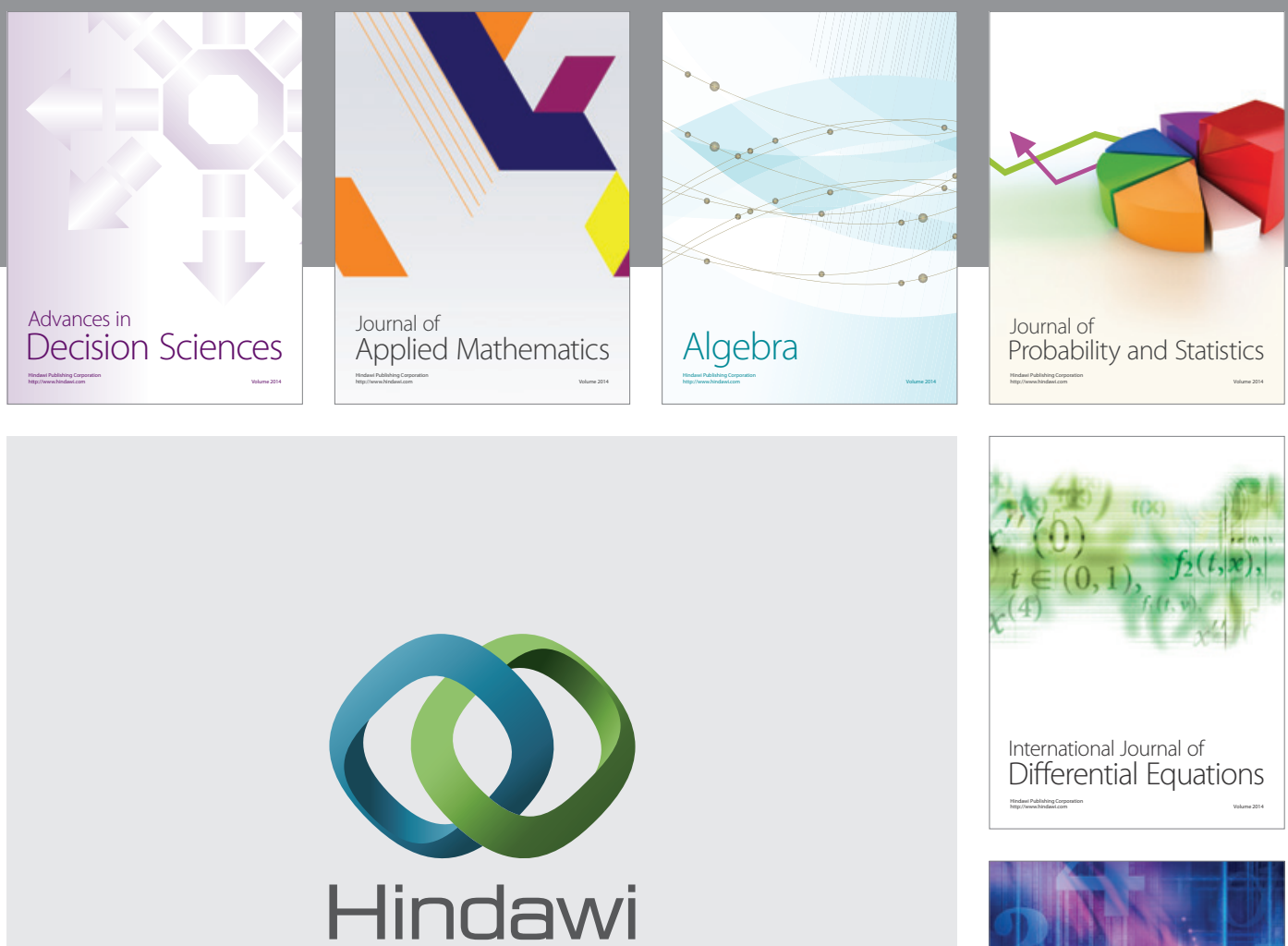

Submit your manuscripts at http://www.hindawi.com
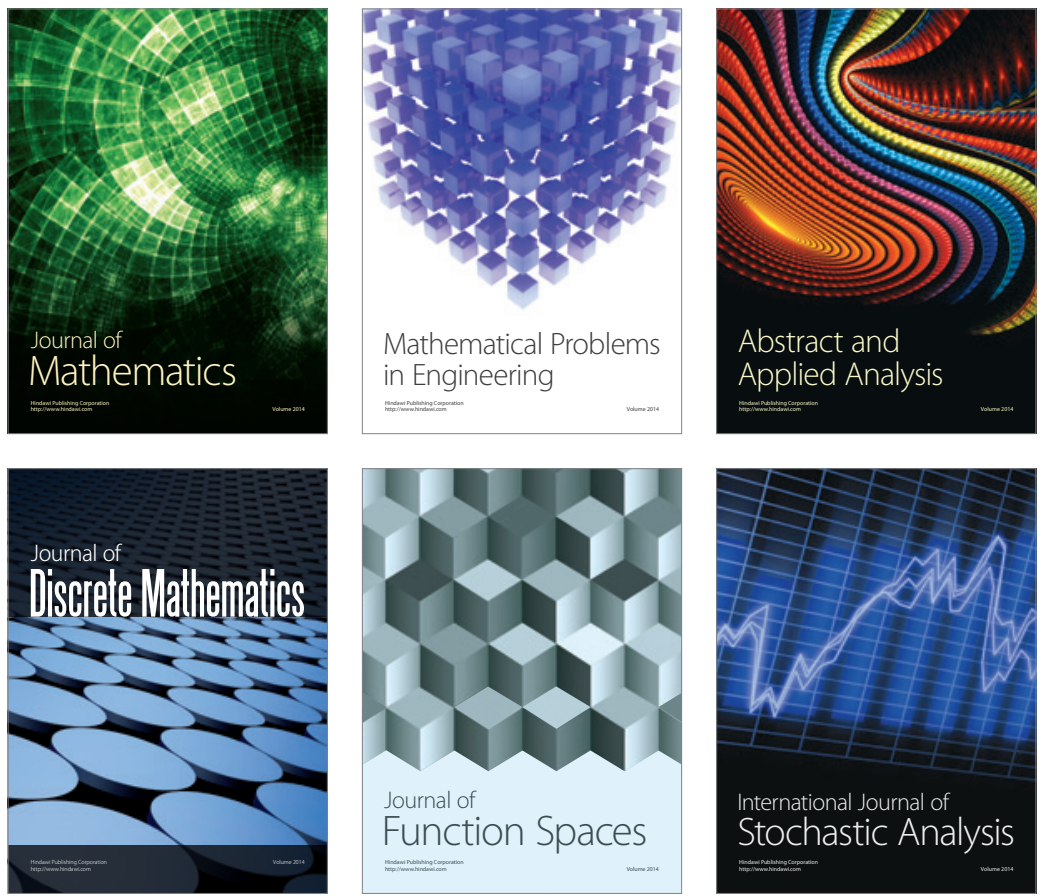

Journal of

Function Spaces

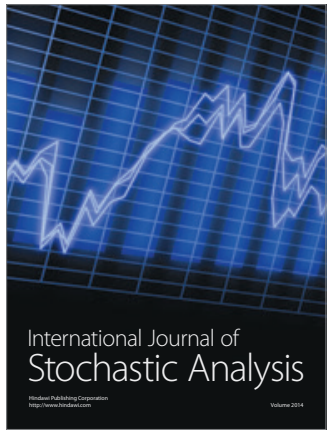

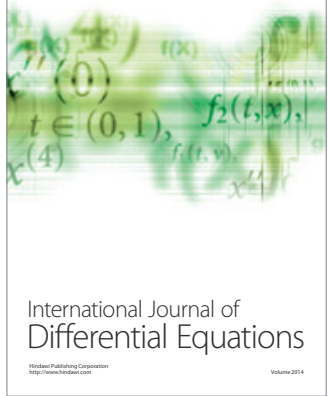
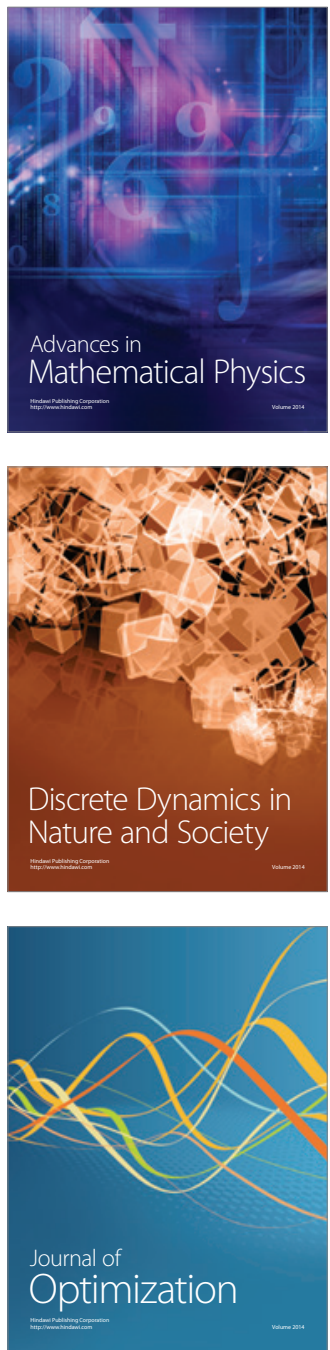